\title{
Mixmaster numerical behavior and generalizations
}

\author{
K. Ferraz and G. Francisco \\ Instituto de Física Teórica (Universidade Estadual Paulista), Rua Pamplona 145, 01405 São Paulo, São Paulo, Brazil
}

(Received 19 August 1991)

\begin{abstract}
The numerical simulation of the mixmaster universe serves the purpose of suggesting two kinds of results. The intrinsic time evolution, during contraction, will be seen to be nonchaotic. This is a necessary feature of relativistic cosmological models undergoing this kind of motion. The mixmaster model also provides a clue on how to define chaoticity for systems described by nonautonomous sets of differential equations.
\end{abstract}

PACS number(s): 98.80.Dr, 04.90. $+\mathrm{e}$

\section{INTRODUCTION}

This paper provides a description on how to use numerical simulations as an aid to foresee proofs of relevant results and to propose ways to quantify the chaotic behavior of nonlinear systems. Our experience relies on the study of the mixmaster evolution, which is rich enough to allow this kind of development. After the pioneering works of Misner [1] and Belinskii, Lifshitz, and Khalatnikov $[2,3]$, the mixmaster universe has been widely studied in many contexts. Here we will focus on its chaotic $[4,5]$ and qualitative behavior [6-8] as well as on numerical simulations [9-13]. More specifically, we intend to present a comprehensive treatment about the decay of the maximal Lyapunov exponent (LE) and, more generally, to propose a definition of chaoticity which applies to sets of nonautonomous ordinary differential equations.

In Sec. II of this work we make some pertinent remarks on the decay of the LE in numerical simulation (such effect was first found in Ref. [9]). This serves as a clue in Sec. III to consistently use analytic and qualitative properties of the system in order to provide conclusive evidence that the mixmaster universe is nonchaotic in $\Omega$ time (defined below). Such analysis if facilitated by the introduction of the information associated with the maximal exponent. By observing the divergence of nearby orbits we are able, in Sec. IV, to identify the essential feature needed to define chaotic behavior, for general systems. Concluding remarks are made in Sec. V.

\section{NUMERICAL EVOLUTION}

The mixmaster system (diagonal, Bianchi type-IX model) derived from Einstein field equations is given by a set of differential equations $[1,2,14]$

$$
\begin{aligned}
a_{i}^{\prime} & =\frac{a_{i} x_{i}}{2}, \\
x_{i}^{\prime} & =\left(a_{j}^{2}-a_{k}^{2}\right)^{2}-a_{i}^{4}
\end{aligned}
$$

and cyclic permutations with $\left(a_{i}, x_{i}\right) \in \mathbb{R}^{6}$, where the motion is constrained by

$$
\sum_{i<j} x_{i} x_{j}=\left(\sum_{i=1}^{3} a_{i}^{2}\right)^{2}-4 \sum_{i<j} a_{i}^{2} a_{j}^{2} .
$$

The scales $a_{1}^{2} a_{2}^{2}, a_{3}^{2}$ represent the diagonal spatial components of the metric tensor defined on a $\mathrm{SO}(3)$-invariant basis and the primes denote derivative with respect to the intrinsic time $d \Omega=-\left(a_{1} a_{2} a_{3}\right)^{-1} d t, t$ being the synchronous time [14].

Combinatorial solutions, approximating true orbits of the system (1)-(3) during contraction $\Omega \rightarrow \infty$, can be obtained by piecing together Bianchi type-I models specified by a sequence of Kasner parameters $\left\{u_{k}\right\}$. On approach to the initial singularity, $k \rightarrow \infty$, one obtains from $\left\{u_{k}\right\}$ an infinite sequence $\left\{x_{n}\right\}$ defining the one-dimensional discrete map $T: x_{n} \rightarrow x_{n+1}$ or bounce transformation. Thus the evolution is coded into the discrete orbit $\left\{T^{n} x_{0}\right\}$ where the exact analytical form of $T$ can be explicitly determined $[2,4]$. Since $T$ is an ergodic and expansive map on the unit interval, it is clear that the discrete orbit has a well-defined positive LE and the discretized system is chaotic. When standard algorithms $[15,16]$ are applied to the calculation of the LE directly from system (1), (2) an entirely different behavior emerges since this exponent tends to decrease as $\Omega \rightarrow \infty$ (the reason for this was explained in Ref. [9]). Such a result is an indication that a chaotic discretization does not always guarantee that the continuous orbit possesses a fixed positive Lyapunov exponent. Considering a solution $v(\Omega)$, with $\left\|v\left(\Omega_{0}\right)\right\|=1$, obtained by linearizing systems (1), (2) in the variables $a_{i}$ and $x_{i}$, the maximal Lyapunov exponent is defined by $\lambda=\lim _{\Omega \rightarrow \infty} \Omega^{-1} \log _{2}\|v(\Omega)\|$. Operationally the Lyapunov exponent is given by [15]

$$
\lambda_{N}=\frac{1}{N} \sum_{n=1}^{N} \lambda_{\mathrm{loc}}\left(\Omega_{n}\right),
$$

where we introduced the local Lyapunov exponent

$$
\lambda_{\text {loc }}\left(\Omega_{n}\right)=\frac{1}{\Omega_{n}-\Omega_{n-1}} \log _{2}\left\|v\left(\Omega_{n}\right)\right\|
$$

and for simplicity we omitted writing in the formulas explicit reference to the initial conditions at the initial time $\Omega_{0}$. Successive redefinitions are made to avoid overflow at each step in such a way [16] that $v\left(\Omega_{n}\right)$ is obtained by propagating $v\left(\Omega_{n-1}\right)$, with $\left\|v\left(\Omega_{n-1}\right)\right\|=1$, during the time $\Omega_{n}-\Omega_{n-1}$. A typical value for $N$ is 3500 corre- 
sponding to $\Omega_{N}=700$ and $\Omega_{n}-\Omega_{n-1}=0.2$. By using a Hamming predictor-corrector algorithm on a micro VAX-II computer, we propagated physical initial conditions [i.e., conditions satisfying (3)] and computed the Lyapunov exponent. Notice that in Fig. 1 the bounces are clearly visible. Using the crucial fact that only the bounces contribute to the effective divergence of the orbits [3], one can infer the following conclusion about the form of the function $\lambda(\Omega)=\Omega^{-1} \log _{2}\|v(\Omega)\|$. During the $n$th Kasner era no divergence occurs and $\log _{2}\|v(\Omega)\|=\alpha_{n}$, for some constant $\alpha_{n}>0$, which means that the LE decays as $\Omega^{-1}$ during each era. Clearly, such argument does not constitute a proof that $\lambda(\Omega)$ is globally of the form $\alpha \Omega^{-1}$ for some $\alpha>0$ and from this one cannot conclude that $\lambda(\Omega) \rightarrow 0$ as $\Omega \rightarrow \infty$.

\section{INFORMATION AND MIXMASTER CHAOTIC BEHAVIOR}

In order to deal with arbitrary systems we found it expedient to define the information $\mathscr{I}(\Omega)$ associated with the maximal Lyapunov exponent. This is given by

$$
\mathcal{J}(\Omega)=\log _{2}\|v(\Omega)\| \text {. }
$$

A relationship between $\mathcal{J}$ and $\lambda_{\text {loc }}$ is easily established by taking $\Omega_{n}-\Omega_{n-1} \rightarrow 0$ in (5):

$$
\frac{d \mathcal{I}(\Omega)}{d \Omega}=\lambda_{\mathrm{loc}}(\Omega)
$$

(compare with Shaw [17], p. 93). As regarding the maximal Lyapunov exponent, one obviously has $\lambda=\lim _{\Omega \rightarrow \infty}(1 / \Omega) \mathscr{I}(\Omega)$. The numerical study of $\mathscr{I}(\Omega)$ is facilitated by the fact that orbits will naturally align in the direction associated with the maximal exponent and our conclusions will not depend on the initial direction $v\left(\Omega_{0}\right)$. In addition, the criterion for chaoticity given below will not depend on the choice of equivalent norms.

As an illustration, we plot $\mathscr{I}(\Omega)$ for the mixmaster model in Fig. 2. The bounce transitions are represented by spikes in this figure followed by increasing eras where no loss of information occurs (compare with Fig. 1). A visual inspection reveals, as expected, that the bounces are responsible for the steady increase of $\mathcal{J}(\Omega)$. We also observe that the bounces tend to become widely dispersed and on the average, one can prove that after the $n$th bounce, and sufficiently close to the singularity, the corresponding amount of elapsed $\Omega$ time is [18]

$$
\Omega_{n} \approx \Omega_{0} e^{n} .
$$

Thus, Fig. 2 clearly exhibits the effect of the discretization and this system could have been classified as chaotic

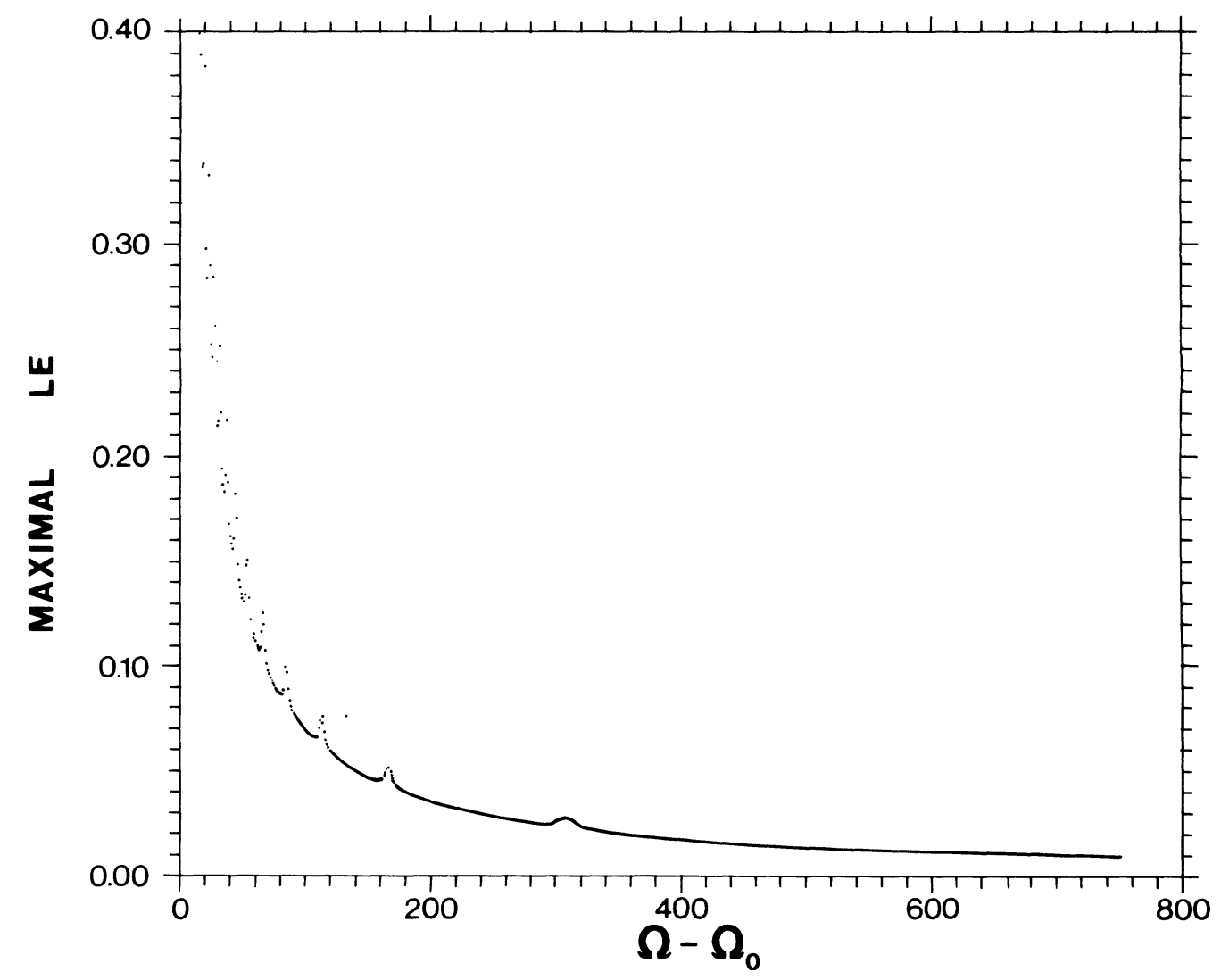

FIG. 1. Our algorithm taken from Ref. [16] calculates all the LE's and was tested against well-known systems (Lorenz, Rosslerchaos, Rossler-hyperchaos). Here is shown the maximum LE for the mixmaster system with physical initial conditions: $a_{1}=1.85400000, a_{2}=0.43850000, a_{3}=0.08540000, x_{1}=-0.36216354, x_{2}=0.517981411, x_{3}=68.44496487$. For a recent, detailed simulation of this model, see the paper by Hobil et al. in Ref. [11]. 


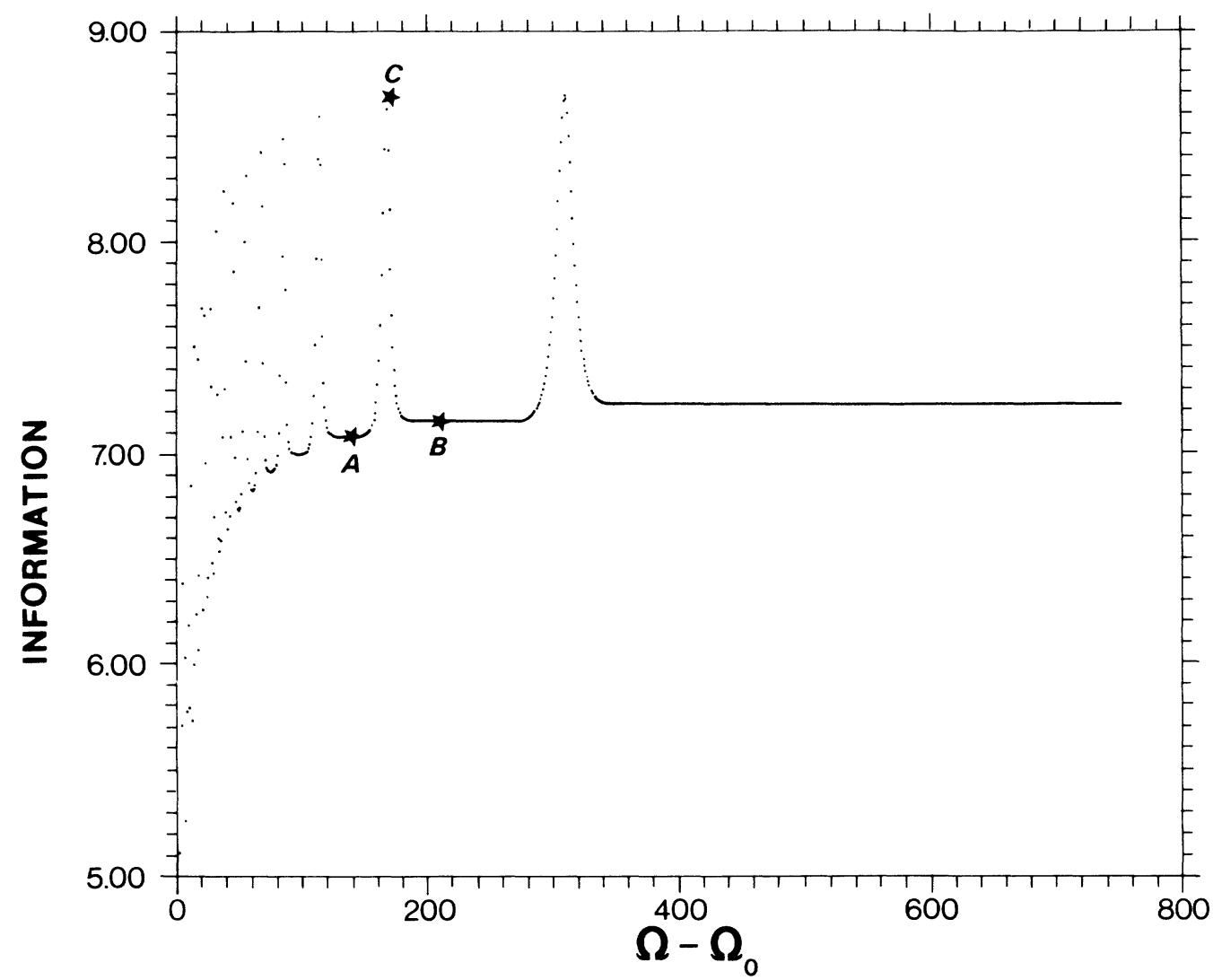

FIG. 2. Same initial conditions as in Fig. 1. The information (6) is shown together with some specific points on its plot: $A, B$, and $C$. The bounces of the discrete map $T$ become more evident in $\Omega$-time evolution as the singularity is approached.

if the average $\Omega$-time distance between bounces were finite. According (8) this is not the case and such a peculiar situation results in the anomalous behavior of the LE.

By disregarding the time elapsed between iterations of the map $T$, one obtains the LE for the discrete orbit. This exponent is just the average information [17] $\langle I\rangle=\lim _{n \rightarrow \infty} n^{-1} \sum_{k=1}^{n} I_{k}$ with $I_{k}=\log _{2}\left|T^{\prime}\left(u_{k}\right)\right|$ or, for $n$ sufficiently large,

$$
\sum_{k=1}^{n} I_{k} \approx n\langle I\rangle .
$$

Here $T$ is defined on the unit interval or, more precisely, on the circle. Local instability of discrete orbits is related to $T^{\prime}$ defined on the tangent space to the circle. According to the Bogoyavlenskii and Novikov qualitative approach [6,7], this circle belongs to an attractor which describes the dynamical behavior of phase-space orbits in the asymptotic region towards the singularity. Then, clearly, the existence of the attractor implies that the instability of $T$ induces the instability of nearby orbits evolving in $\Omega$ time. This means that, in analogy with (9), the average information $\langle\mathcal{J}\rangle$ associated with the continuous orbit evolution is finite. Then, after $n$ eras, we have

$$
\sum_{k=1}^{n} \mathcal{J}_{k} \approx n\langle\mathcal{I}\rangle,
$$

where $\mathscr{J}_{k}$ represent net increases of $\mathscr{I}(\Omega)$ during the bounces (e.g., $\mathfrak{J}_{B}-\mathfrak{J}_{A}$ in Fig. 2). The left-hand side of (10) is the total loss of information after $n$ bounces in $\Omega$ time. This corresponds to $\mathcal{J}\left(\Omega_{n}\right)$ which, from (10), is just, $\mathscr{I}\left(\Omega_{n}\right) \approx n\langle\mathscr{J}\rangle$. Thus, using (8), the general conclusion is that

$$
\mathcal{J}(\Omega) \approx \text { const } \times \log \Omega .
$$

Consequently, from (6), divergence is no faster than polynomial and the system is nonchaotic in $\Omega$ time (a similar argument shows that the system is chaotic with respect to the synchronous time evolution [19]).

\section{NONAUTONOMOUS SYSTEMS}

Here we intend to provide a characterization of chaotic behavior for nonautonomous systems $x^{\prime}=f(x, \tau), \tau$ some time scale, where the explicit time dependence of the smooth function $f$ constantly changes the form of the equation. (The mixmaster universe is a particular example since its Hamiltonian is time dependent [1].) We expect that in such systems the LE will not converge to a constant value on finite-time intervals as in autonomous systems and, in this sense, $\lambda$ will be time dependent.

Our main argument will be based on Fig. 2 where, during a bounce, the divergence of orbits reaches a maximum at point $C$ but the net loss of information is given by $\mathcal{I}(B)-\mathcal{I}(A)$ in that portion of the diagram. A more drastic model divergence can be imagined by taking $\|v(\tau)\|=\exp \left(\tau \sin ^{2} \tau\right)$, where evolution occurs for $\tau \rightarrow \infty$. 
In this case the minimum value of the information always returns to zero, while the maximum increases linearly. In searching for a broad definition of chaoticity one must consider that for time-dependent LE's much more varied kinds of behaviors are, in principle, allowed other than $\|v(\tau)\| \approx \exp (\lambda \tau)$ found in chaotic autonomous systems. Using the mixmaster as a guide it is not difficult to pinpoint the essential feature needed to formulate a viable generalization: the returns of $\mathcal{J}$. For example, if this quantity always returns to zero, no matter what happens in the diverging phases such behavior cannot be considered chaotic. In general we introduce $\mathscr{H}(\tau)$ given by

$$
\mathscr{H}(\tau)=\inf _{\omega \geq \tau} \mathcal{J}(\omega)
$$

where $\mathcal{I}$, as before, is the information associated with the maximal LE. Then define the limit

$$
\Lambda=\lim _{\tau \rightarrow \infty} \frac{\mathscr{H}(\tau)}{\tau} \text {. }
$$

Evidently the infimum can be dropped when studying autonomous systems and in this case $\Lambda$ coincides with the usual maximal LE. For time-dependent LE's there are several other possible types of behaviors and the infimum is essential. According to these ideas, and considering only positive information, it is natural to propose the following classification. A chaotic system satisfies $\Lambda>0$ (for almost all initial conditions in some measure theory sense). Systems for which $\Lambda=\infty$ should also be considered chaotic (e.g., $\|v(\tau)\| \approx \exp [\exp (\tau)], \exp (\tau \log \tau)$, etc. ). An interesting situation occurs when $\Lambda$ vanishes. If $\Lambda=0$ and $\lim _{\tau \rightarrow \infty} \mathscr{H}(\tau) / \log \tau=c \geq 0$ then the divergence of orbits is not faster than the polynomial and the system is nonchaotic. On the other hand, we could have $\Lambda=0$ and $\lim _{\tau \rightarrow \infty} \mathcal{H}(\tau) / \log \tau=\infty$. This happens whenever local divergence assumes the form $\exp (\sqrt{\tau})$, $\exp \left(\log ^{\alpha} \tau\right), \alpha>1$, etc. We avoid here making any statement about sensitive dependence on initial conditions associated with these functions. However, since they grow faster than any polynomial, we suggest calling such systems weakly chaotic. The mixmaster dynamical evolution
[1] already indicates that weakly chaotic behavior might be found in the class of expanding wall billiards for specific values of the wall speed.

\section{CONCLUSIONS}

According to the Belinskii-Lifshitz-Khalatnikov paradigm [3], Bianchi type-IX oscillatory behavior will be found in a large class of relativistic cosmological systems evolving towards the initial singularity. At least for homogeneous models we have shown that such evolution is nonchaotic in $\Omega$ time. Probably this result is likely to hold in more general contexts. Another relevant fact is that this system is chaotic with respect to the synchronous time evolution $[12,19,20]$ where the singularity is approached as $t \rightarrow 0^{+}$. This means that chaos is a concept which depends on which time scale is used to parametrize the motion. The methods and theorems of dynamical systems theory [6-8] normally refer to some time parameter which grows unboundedly. This is the case where the generalizations in Sec. IV apply although general relativity is a multiple-time-scale theory and the synchronous time is, in principle, as good as any other choice. Thus the existence of a chaotic discretization in relativistic cosmological models does not guarantee that the system is chaotic for every time scale. Its existence is an indication that it should be possible to find at least one time scale according to which the system is chaotic. In addition, it is interesting to note that although the equations which define the mixmaster evolution are reversible, the discretization is not (see Burd, Buric, and Tavakol in Ref. [21]).

\section{ACKNOWLEDGMENTS}

The authors would like to thank Dr. George Matsas for many invaluable discussions. Financial support was partially provided by Coordenação de Aperfeiçoamento de Pessoal de Nivel Superior (K.F.) and Conselho Nacional de Desenvolvimento Cientifico e Tecnológico (G.F.).
[1] C. Misner, Phys. Rev. 186, 1319 (1969); 186, 1328 (1969); Phys. Rev. Lett. 22, 1071 (1969).

[2] V. A. Belinskii, E. M. Lifshitz, and I. M. Khalatnikov, Adv. Phys. 19, 525 (1970).

[3] V. A. Belinskii, E. M. Lifshitz, and I. M. Khalatnikov, Adv. Phys. 31, 639 (1982).

[4] J. D. Barrow, Phys. Rev. Lett. 46, 963 (1981); Phys. Rep. 85, 1 (1982).

[5] D. F. Chernoff and J. D. Barrow, Phys. Rev. Lett. 50, 134 (1983).

[6] O. I. Bogoyavlenskii and S. P. Novikov, Sel. Math. Sov. 2, 159 (1982).

[7] O. I. Bogoyavlenskii, Qualitative Theory of Dynamical Systems in Astrophysics and Gas Dynamics (Springer-Verlag,
Berlin, 1985).

[8] P. K-H.-Ma and J. Wainwright, presented at the Proceedings of the Third Hungarian Relativity Workshop, 1989 (unpublished).

[9] G. Francisco and G. E. A. Matsas, Gen. Relativ. Gravit. 20, 1047 (1988).

[10] A. B. Burd, N. Buric, and G. F. Ellis, Gen. Relativ. Gravit. 22, 349 (1990).

[11] D. Hobil, D. Bernstein, M. Welge, and D. Simkins, Class. Quantum Grav. 8, 1155 (1991).

[12] S. E. Rugh and B. J. T. Jones, Phys. Lett. A 147, 353 (1990).

[13] B. K. Berger, Class. Quantum Grav. 7, 203 (1990).

[14] L. D. Landau and E. M. Lifshitz The Classical Theory of 
Fields (Pergamon, Oxford, 1975).

[15] G. Benettin, L. Galgani, and J. M. Strelcyn, Phys. Rev. A 14, 2338 (1976).

[16] A. Wolf, J. B. Swift, H. L. Swinney, and J. A. Vastano, Physica 16D, 285 (1985).

[17] R. Shaw, Z. Naturforsch 36a, 80 (1981).

[18] I. M. Khalatnikov, E. M. Lifshitz, K. M. Khanin, L. N. Schur, and Ya G. Sinai, J. Stat. Phys. 38, 97 (1985).
[19] K. Ferraz, G. Francisco, and G. E. A. Matsas, Phys. Lett. A 156, 407 (1991).

[20] J. Pullin, presented at the Proceedings of the VII Latin American Symposium on Relativity and Gravitation, Mexico, 1990 (unpublished).

[21] A. B. Burd, N. Buric, and R. K. Tavakol, Class. Quantum Grav. 8, 133 (1991). 